\title{
Cosmic results with the final Micromegas sectors for the ATLAS Muon upgrade
}

\author{
Gabriel Rabanal Bolaños*, on behalf of the ATLAS Muon Collaboration \\ Department of Physics, Harvard University, \\ 17 Oxford Street, Cambridge, MA 02138, United States \\ E-mail: gabriel.rabanal.bolanos@cern.ch
}

The detector technology for the innermost end-cap muon stations (Small Wheels) of the ATLAS detector needs to be upgraded to cope with the upgraded beam luminosity in the High Luminosity Large Hadron Collider (HL-LHC). The new technology should be able to meet the demands of high efficiency and fast response at the expected high background rate. The detectors for precision tracking and triggering at the New Small Wheel (NSW) will be Micromegas (MM) and small-strip Thin Gap Chambers (sTGC). The detectors are also complementary to each other. Each of the two NSW will consist of 8 large and 8 small sectors. A sector is a combination of the sTGC wedges on either side of a double MM wedge. The MM quadruplets are received at CERN from different construction sites. $4 \mathrm{MM}$ quadruplets are integrated to build a MM double wedge. After the electronic integration is completed, the double wedges are tested with cosmic muons at the Cosmic Stand of B899 at CERN. There is a sequence of procedures for testing the double wedges at the Cosmic Stand. Here, we make sure of the final high voltage configuration, measure the efficiency, cluster size, strip multiplicity per readout layers of the double wedge, and qualify the MM sector for the final integration with the sTGC wedges before mounting them on the NSW. The procedure and the final results from the MM double wedges will be presented.

40th International Conference on High Energy physics - ICHEP2020

July 28 - August 6, 2020

Prague, Czech Republic (virtual meeting)

\footnotetext{
${ }^{*}$ Speaker
} 


\section{Introduction}

The Large Hadron Collider (LHC) will undergo a major upgrade, the High Luminosity LHC (HL-LHC) [1] which will increase its instantaneous luminosity by a factor of five with respect to the original design value, up to $7.5 \times 10^{34} \mathrm{~cm}^{-2} \mathrm{~s}^{-1}$. For this purpose, the end-cap muon stations of the ATLAS experiment [2], the Small Wheels (SW) (Figure 1) will need to be upgraded to cope with this increased hit rate.

An analysis of 2012 data demonstrates that approximately $90 \%$ of the muon triggers in the end-caps are fake [3], so the upgrade must also be able to eliminate these fake triggers. To satisfy the requirement on the muon $p_{\mathrm{T}}$ resolution of $10 \%$ at

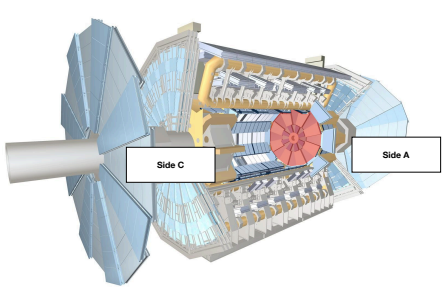
$1 \mathrm{TeV}$, the upgrade needs a spatial resolution of $100 \mu \mathrm{m}$ or better.

Figure 1: Diagram of the ATLAS detector, with the SWs highlighted in red.

\section{New Small Wheel (NSW)}

The NSW consists of 16 sectors: 8 small modules (SM) and 8 large modules (LM), see Figure 2. Each sector contains two technologies: resistive-strip Micromegas (MM) detectors for precision tracking and small-strip Thin Gap Chambers (sTGC) for triggering capabilities, with the following configuration: sTGC-MM-MM-sTGC. The NSW MM detectors will cover an active area of $1280 \mathrm{~m}^{2}$.

The principle of operation of the MM technology is illustrated in Figure 3. Each MM layer is a parallel plate gaseous detector. The drift cathode is kept at $-300 \mathrm{~V}$. The drift region between the cathode and the thin metal mesh is filled with a mixture of $\mathrm{Ar}+7 \% \mathrm{CO}_{2}$ where free drift electrons caused by gas ionization can move in the electric field and pass through the mesh, which is kept at ground. The region between the mesh and the readout electrodes is the amplification region, and the voltage there is kept at $+570 \mathrm{~V}$. The resultant electric field is a much stronger electric field $(\sim 75$ times stronger) than the one in the drift region, so the electrons that pass through the mesh then produce avalanches in the amplification region. The avalanches then induce signals on the readout strips. According to the NSW Technical Design Report (TDR) [3], a MM layer is able to track particles with a precision of the order of $100 \mu \mathrm{m}$ per detection gap while withstanding a particle rate up to $\sim 20 \mathrm{kHz} / \mathrm{cm}^{2}$.

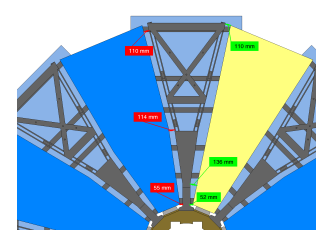

(a)

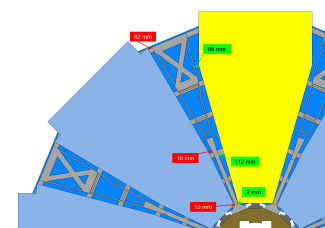

(b)

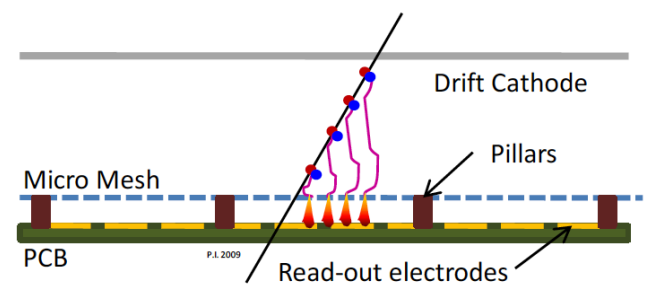

Figure 2: Layout of the different sizes of sectors in the NSW, showing (a): Small Modules, (b): Large Modules. [3]

Figure 3: Operating principle of a Micromegas layer. [3] 
A complete description of the NSW can be found in Ref. [3].

\section{Cosmic Ray Test Stand}

The cosmic test stand of Building 899 at CERN (BB5) is used to complete the final electronics integration of each MM chamber. Here, the data acquisition system (DAQ) is tested, and measurements are taken regarding the noise rate, $\mathrm{HV}$, gas leaks, spark rate, efficiency, and an estimation of the space resolution and time resolution using cosmic muons. In the cosmic stand, pairs of scintillators are used to make a trigger with a frequency of $\approx 100 \mathrm{~Hz}$ (Figure 4).
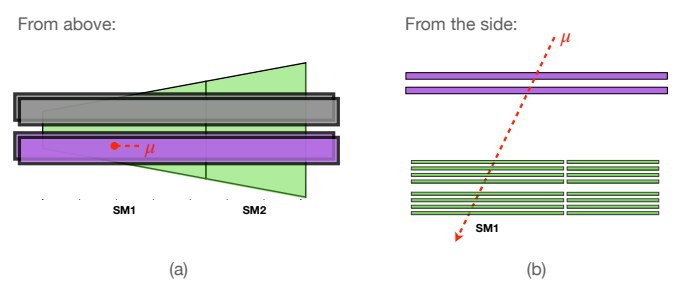

Figure 4: Setup of the Cosmic Ray Test Stand at Building BB5 at CERN.

\section{Noise}

The noise in a MM chamber is measured in dedicated runs (not during data taking) by delaying the singles signal from one scintillator in order to avoid cosmic particles. The hardware threshold has to be set low enough to get a signal and high enough to keep the noise below the predicted hit rate at the HL-LHC, which is $1 \mathrm{kHz} / \mathrm{strip}$. The baseline noise varies from strip to strip, and the root mean square (RMS) in each strip is of the order of $\sim 2.5 \mathrm{mV}$. The threshold used for the cosmic studies at BB5 is set to RMS $\times 9$ in each strip, chosen to make the noise be well below the expected hit rate at the HL-LHC. The effective charge threshold is of the order of $\sim 2.5 \mathrm{fC}$.

\section{Tracking}

There are two types of Micromegas layers: eta layers that measure pseudorapidity $(\eta)$ in their precision coordinate, and stereo layers with strips tilted 1.5 degrees (with respect to the strips of eta layers) to gather azimuthal angle information $(\phi)$. A simple clustering algorithm groups neighboring hit strips into clusters used to fit tracks. Clusters are defined with at most two consecutive holes. For vertical tracks we expect clusters to contain three strips, on average. The mean cluster charge and cluster width are shown in Figures 5 and 6. These figures show cosmic data taken with an eta SM layer and a stereo SM layer, and with two data-taking configurations:

- Neighbors off: The signal in a strip is read only if it is above the signal threshold (RMS $\times 9$ )

- Neighbors on: If the signal in a strip is above the signal threshold (RMS $\times 9)$, then it is read and also the adjacent strips are read even if they are below the threshold.

In Figure 6 the observed cluster length is compared to the geometrical projection, calculated assuming that a track incident at an angle $\theta$ will induce a signal in all the strips projected from the track segment contained in the drift region. The formula is $1+\tan \theta \cdot h_{\text {drift gap }} /$ pitch where $h_{\text {drift gap }}$ is the height of the drift gap $(5 \mathrm{~mm})$ and the pitch is $0.425 \mathrm{~mm}$ for SM chambers. 


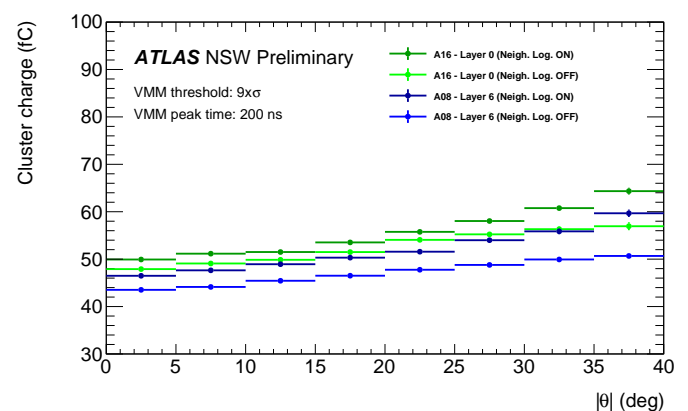

Figure 5: Mean Micromegas cluster charge vs. incident angle $\theta$ (deviation from a straight vertical track) for eta layers. [4]

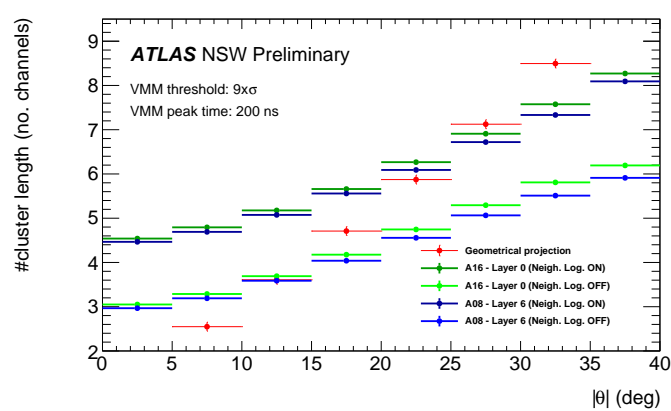

Figure 6: Mean number of strips per cluster vs. incident angle $\theta$ for MM eta layers. The geometrical projection is: $1+\tan \theta \cdot h_{\mathrm{drift} \text { gap }} /$ pitch. [4]

\section{High Voltage Validation}

Each NSW MM layer is divided into 16 high voltage (HV) sections which means that each MM sector (8 layers) has $128 \mathrm{HV}$ sections. The TDR initially had $600 \mathrm{~V}$ as the nominal amplification high voltage [3], but the new nominal high voltage has been set to $570 \mathrm{~V}$ due to sparking in multiple HV sections. HV instabilities have been observed in all the first full-size MM prototypes. For 7 small chambers tested in the cosmic ray test stand (a total of $896 \mathrm{HV}$ sections):

- $803 \mathrm{HV}$ sections $(89.6 \%)$ could hold the nominal high voltage of $570 \mathrm{~V}$

- $80 \mathrm{HV}$ sections (8.9\%) supported between $500 \mathrm{~V}$ and $560 \mathrm{~V}$

- $13 \mathrm{HV}$ sections (1.5\%) could not support $500 \mathrm{~V}$ and had to be turned off.

This is not a problem for tracking as long as the sections with lower-than-optimal HV do not overlap in towers on top of each other. If one HV section has low voltage or is turned off due to sparking, there are other 7 remaining layers that can do tracking. There is no case where two sections are off and in the same tower. The mean cluster charge and mean cluster width as a function of the applied amplification HV, for all incident angles, are shown in Figures 7 and 8.

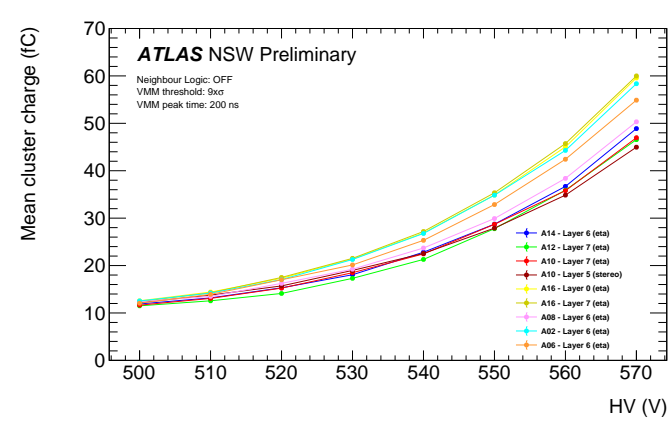

Figure 7: Mean Micromegas cluster charge vs. amplification high voltage. [4]

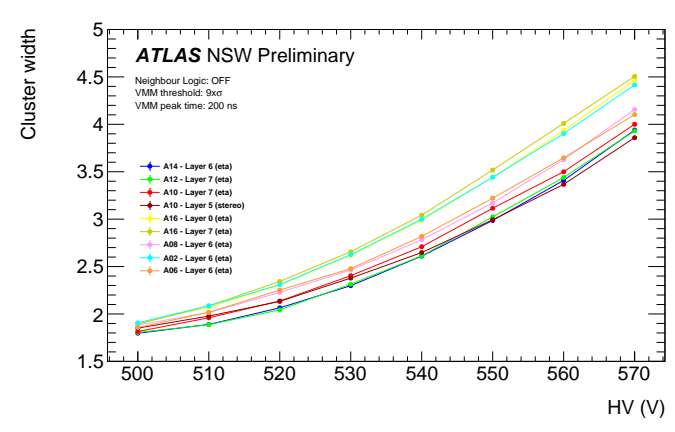

Figure 8: Micromegas cluster width (total number of strips per cluster) vs. amplification high voltage. [4] 


\section{Efficiency}

The efficiency in each layer is evaluated using the tag and probe method in each layer: A tag is defined as a track reconstructed using at least 6 other layers of the chamber. A probe is defined as a cluster whose charge-weighted centroid is near within a window with respect to the extrapolated track position in the layer. The window is $\pm 5 \mathrm{~mm}$ for the eta layers and $\pm 10 \mathrm{~mm}$ for the stereo layers. The number of probes divided by the number of tags is the efficiency. For the layers that could hold an amplification $\mathrm{HV}$ of $570 \mathrm{~V}$, their efficiency was measured while decreasing the voltage in steps of $10 \mathrm{~V}$. The results for several Micromegas layers are shown in Figure 9.

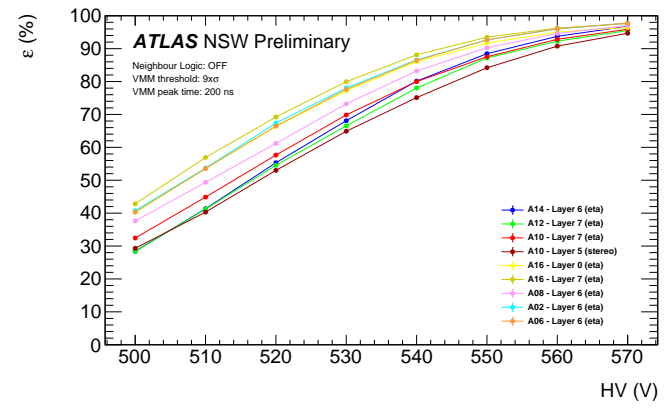

Figure 9: Micromegas efficiency vs. amplification high voltage for several SM chamber layers tested with cosmic muons at BB5. [4]

\section{Conclusion}

The final MM chambers of the NSW for the ATLAS detector are being tested with a cosmic muon test stand at CERN. 7 out of the 16 planned SM chambers had a complete electronics integration and were tested. No LM chamber has been tested yet. It was determined how many sections of each SM chamber could hold nominal amplification HV $(89.6 \%)$ and the efficiency of each layer was then measured as a function of HV. The operating gas was a mixture of $\mathrm{Ar}+7 \%$ $\mathrm{CO}_{2}$. The spatial resolution of the chambers is a work in progress and needs further investigation. As per the latest NSW Review meeting on 20 July 2020, the commissioning of the NSW Side A is projected to be finished by May 2021 and Side C by October 2021, both ready for use for the upcoming LHC Run 3 and HL-LHC data-taking periods.

\section{Acknowledgements}

This material is based upon work supported by the U.S. Department of Energy, Office of Science, Office of Basic Energy Sciences under Award Number DE-SC-0007881.

\section{References}

[1] Apollinari G., Béjar Alonso I., Brüning O., Fessia P., Lamont M., Rossi L. and Tavian L. High-Luminosity Large Hadron Collider (HL-LHC): Technical Design Report V. 0.1, CERN-2017-007-M, https://cds.cern.ch/record/2284929.

[2] ATLAS Collaboration, The ATLAS experiment at the CERN Large Hadron Collider, 2008 JINST 3 S08003.

[3] ATLAS Collaboration, New Small Wheel Technical Design Report, CERN-LHCC-2013-006, ATLAS-TDR-020. 
[4] ATLAS Collaboration, NSW Public Results,

https://twiki.cern.ch/twiki/bin/view/AtlasPublic/NSWPublicResults. 